\title{
Importance Evaluation of Power Communication Network Node Based on FCM Clustering
}

\author{
Qiao Xue*, Huixia Ding and Xiangzhou Chen \\ No. 15 Xiaoying East Road, China Electric Power Research Institute, Qinghe, Beijing 100192 China \\ ${ }^{*}$ Corresponding author
}

\begin{abstract}
The node importance evaluation of network is of great significance for the improvement of power communication network's reliability. At present, the indexes used in the node importance evaluation in power communication networks are usually one - sided. Dealing with this, this paper presents a multiindex based node importance evaluation method. In this method, degree centrality, betweenness centrality and eigenvector centrality are employed as indexes, and fuzzy clustering method is adopted to classify network nodes, to finally obtain the set of important nodes. The method can be applied to the evaluation of node importance in power communication network. The experiment reflects the effectiveness of the method.
\end{abstract}

Keywords-power communication network; node importance; fuzzy clustering

\section{INTRODUCTION}

Power communication network is the communication network of the power system. It carries all the services of power dispatching, management and production, which is an important part of the power grid. With the construction of smart grid, power communication network carrying the business and the scale continues to expand. The power system is also becoming more and more dependent on the power communication network, so it can be said that the normal operation of power communication network is stable [1]. To a large extent, the important nodes affect the stability and reliability of power communication network. Power communication network is a typical complex network system [2]. Research shows that in scale-free networks, when $5 \%$ of the core nodes are attacked, the network will be paralyzed[3]. Therefore, it is of great help to improve the reliability of the network by accurately excavating the important nodes in the network.

Compared with the importance evaluation of the network edges, the importance evaluation of nodes in the network has not been paid much attention, and the existing evaluation methods are relatively limited [4]. At present, the network node importance evaluation mainly uses the degree method [7], betweenness method [5], node deletion method [6], node contraction method [7], and PageRank algorithm [8]. However, each evaluation method has a certain one-sidedness. Using the degree as evaluation index, for example, that the greater the degree of node, the more important the node is, but this method has one-sidedness, some important nodes such as node "bridge" do not necessarily have a larger degree. Node betweenness method does not consider well the influence of nodes on LAN. Node deletion method, analyzes node importance by comparing the change of network connectivity before and after deleting nodes. But if you delete the nodes attached to the end node with a degree of 1 , it is difficult to realize an objective importance evaluation of nodes. Although all the above algorithms reflect node importance to some extent, they cannot accurately describe the importance of nodes in the whole network.

In order to be evaluate the node importance of electric power communication networks in a more objective way, this article uses degree centrality, betweenness centrality and eigenvector centrality as indexes, to evaluate the importance of network nodes through a variety of comprehensive indexes. It realizes the classification of node importance of electric power communication networks by using FCM clustering method. The result shows that this method can effectively evaluate the importance of single nodes in different types of complex networks.

\section{THEORETICAL BASIS}

The power communication network is represented by the graph $\mathrm{G}=(\mathrm{V}, \mathrm{E})$, which is a non-directional loop network, where $\mathrm{V}=\{\mathrm{v} 1, \mathrm{v} 2, \ldots \mathrm{Vn}\}$ is the set of all nodes of the network. $E=\left\{\mathrm{e}_{1}\right.$, $\left.e_{2}, \ldots E_{m}\right\}$ is the set of the edges between nodes, $|E|=m$.

The importance of the network nodes has a certain relationship not only with the degree of the node itself, but also with the importance of the neighbor nodes, these indexes reflect the influence of nodes. The influence of nodes includes direct influence and indirect influence. Direct influence reflects the node's own ability to affect other nodes, such as the degree of the node. Indirect influence reflects the node's ability of to influence other nodes through the network, such as the number of interfaces. In this paper, the degree centrality, betweenness centrality and eigen vector centrality are employed as indexes to evaluate the node importance.

(1) Degree Centrality: connection degree $k$ is defined as the number of edges attached to the node, which reflects the topology characteristics of the network. It shows the node's direct influence on other nodes in the network. In a network with $\mathrm{n}$ nodes, the nodes cannot not exceed n-1. Expression of degree centrality:

$$
\mathrm{DC}_{i}=\frac{k_{i}}{n-1}
$$

where $\mathrm{k}_{\mathrm{i}}$ represents variables associated with node $\mathrm{I}$ in the network.

(2) Betweenness Centrality: node betweenness is defined as the ratio of the number of paths that pass through the point to 
the total number of the shortest paths in all the shortest paths in the network. The betweenness of each node is represented by parameter $\mathrm{Bi}$, which characterizes the ability of node i to control the transmission of information along the shortest path between nodes in a network. The betweenness of the node I is defined as the number of nodes:

$$
B C_{i}=\sum_{s \neq i \neq j} \frac{n_{s t}^{i}}{g_{s t}}
$$

Among them, $g_{s t}$ is the number of shortest paths from node s to node $\mathrm{t}$, and $n_{s t}^{i}$ is the number of the shortest path through node $\mathrm{i}$ in the shortest path of $\mathrm{g}_{\mathrm{st}}$ from node $\mathrm{s}$ to node $\mathrm{t}$.

(3) Eigenvector Centrality: its basic idea is that the importance of a node depends on the number of its neighbors and the importance of its neighbors. Remember that $\mathrm{Xi}$ is the measure of importance of nodes, so there are

$$
x_{i}=c \sum_{j=1}^{n} a_{i j} x_{j}
$$

Where $c$ is a proportional constant and $A=\left(a_{i j}\right)$ is the adjacency matrix of the network. Let $\mathrm{x}=\left[\mathrm{x}_{1}, \mathrm{x}_{2}, \ldots, \mathrm{x}_{\mathrm{n}}\right]^{\mathrm{T}}$, then the above formula can also be written as follows:

$$
\xi=\chi \mathrm{A} \xi
$$

Equation (4) means that $\mathrm{x}$ is the eigenvector of matrix $\mathrm{A}$ corresponding to the eigenvalue $\mathrm{c}^{-1}$.

\section{FCM CLUSTERING}

Fuzzy clustering analysis is a method of using fuzzy mathematics to distinguish objects with similar properties and to classify them. FCM algorithm is one of the fuzzy clustering method is more important, the method of clustering results with each data point membership degree of cluster center, said the membership degree is used to represent a number.

\section{A. FCM Basic Theory}

FCM is an iterative optimization algorithm that divides $n$ vector xi $(\mathrm{I}=1,2, \ldots \ldots \mathrm{N})$ into $\mathrm{C}$ fuzzy clusters, and the clustering center of each cluster is obtained to minimize the objective function, and the objective function of FCM is defined as:

$$
\mathrm{J}(\mathrm{U}, \mathrm{V})=\sum_{k=1}^{n} \sum_{j=1}^{c} u_{i k}^{m} d\left(x_{k}, v_{i}\right)
$$

Among them, $\quad \sum_{i=1}^{c} u_{i k}=1, u_{i k} \in(0,1), d\left(x_{k}, v_{i}\right)=$ $\left\|x_{k}-v_{i}\right\|^{2}$, m stands for weighted index, its value range is $1<\mathrm{m}<+\infty$. The FCM algorithm is different from the $\mathrm{C}$ mean algorithm, which adds the fuzzy weight index $\mathrm{m}$ in the target function. It is an important parameter in FCM, which affects the fuzzy degree of fuzzy clustering results. To minimize the target function, the update method of clustering center and membership function is as follows:

$$
\begin{gathered}
v_{i}=\sum_{k=1}^{n} u_{i k}^{m} x_{k} / \sum_{k=1}^{n} u_{i k}^{m}(i=1,2, \ldots, c) \\
u_{i k}=1 / \sum_{j=1}^{c}\left(\frac{d_{i k}}{d_{j k}}\right)^{1 /(m-1)}(i=1,2, \ldots c ; k=1,2, \ldots, n)(7)
\end{gathered}
$$

\section{B. Algorithm Steps}

In the node importance evaluation of power communication network, this paper divides the nodes into three categories: core node, important node and common node, that is, the number of categories $c=3$. In order to classify fuzzy clustering, the value of fuzzy degree index $m=2$ [9] [10]; the iteration termination threshold $\varepsilon=0.001$. The FCM algorithm is a simple iterative process that takes the following steps to determine the clustering center $\mathrm{V}$ and the membership matrix $\mathrm{U}$.

1) From the $n$ data sets $X=\left(x_{1}, x_{2}, . ., x_{n}\right)$, the initial clustering centers $\mathrm{V}^{0}=\left(\mathrm{v}_{1}, \mathrm{~V}_{2}, \ldots, \mathrm{v}_{\mathrm{c}}\right)$ and the threshold $\varepsilon>0$ are randomly selected.

2) Repeat the following operation, the algorithm stops when $\left\|\mathrm{V}^{\mathrm{b}+1}-\mathrm{V}^{\mathrm{b}}\right\|<\varepsilon$, otherwise $\mathrm{b}=\mathrm{b}+1$ will continue to perform the following steps. $\|\bullet\|$ is the F-norm of the matrix, $\| \mathrm{V}^{\mathrm{b}+1}$ $\mathrm{V}^{\mathrm{b}} \|_{\mathrm{F}}=\sqrt{\sum_{i=1}^{m} \sum_{j=1}^{c}\left(p_{i j}^{b}-p_{i j}^{b+1}\right)^{2}}$.

a) Using the current $\mathrm{V}^{\mathrm{b}}$, calculate the membership matrix $\mathrm{U}^{\mathrm{b}}$ using formula (7).

b) Using the current $\mathrm{U}^{\mathrm{b}}$, calculate the clustering center $\mathrm{V}^{\mathrm{b}+1}$ using formula (6).

\section{EXPERIMENTAL RESULTS}

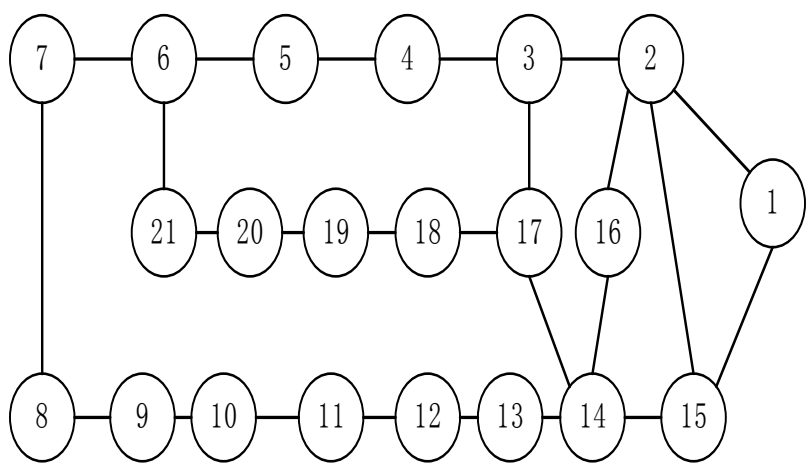

FIGURE I. ARPA NETWORK TOPOLOGY

In order to effectively illustrate the effectiveness of the method in this paper, this paper uses the American ARPA network topology in figure I, which consists of 21 nodes and 26 links. ARPA network topology is the main network topology that is commonly used when analyzing the importance of network nodes. The average value of network is 2-3, and the value of most nodes is 2 .

Table I shows the ordering results of node importance based on literature [11], literature [12] and literature [13]. Three algorithms of node important degree of sorting is slightly different, that is because different methods has different focuses on index selections as well as algorithms, which well testifies the existence of one-sidedness in each algorithm. 
TABLE I. TABLE I IMPORTANT RANKING RESULTS OF ARPA NETWORK NODES

\begin{tabular}{|c|c|c|c|}
\hline \multirow[t]{2}{*}{ ID } & \multicolumn{3}{|c|}{ Ranking } \\
\hline & literature 11] & literature 12] & literature [13] \\
\hline 1 & 21 & 12 & 3 \\
\hline 2 & 9 & 1 & 14 \\
\hline 3 & 1 & 2 & 2 \\
\hline 4 & 6 & 11 & 15 \\
\hline 5 & 8 & 15 & 12 \\
\hline 6 & 4 & 13 & 19 \\
\hline 7 & 13 & 18 & 16 \\
\hline 8 & 17 & 20 & 17 \\
\hline 9 & 16 & 21 & 6 \\
\hline 10 & 12 & 19 & 1 \\
\hline 11 & 10 & 16 & 13 \\
\hline 12 & 2 & 10 & 18 \\
\hline 13 & 7 & 9 & 4 \\
\hline 14 & 5 & 4 & 5 \\
\hline 15 & 19 & 3 & 11 \\
\hline 16 & 20 & 6 & 20 \\
\hline 17 & 18 & 5 & 7 \\
\hline 18 & 11 & 7 & 10 \\
\hline 19 & 3 & 8 & 21 \\
\hline 20 & 14 & 14 & 9 \\
\hline 21 & 15 & 17 & 8 \\
\hline
\end{tabular}

Figure II shows the distribution of the various factors in this paper. It can be seen from the figure that the three relationships are not completely positive correlations. Therefore, considering these three factors, we can avoid the shortcomings of single factor evaluation.

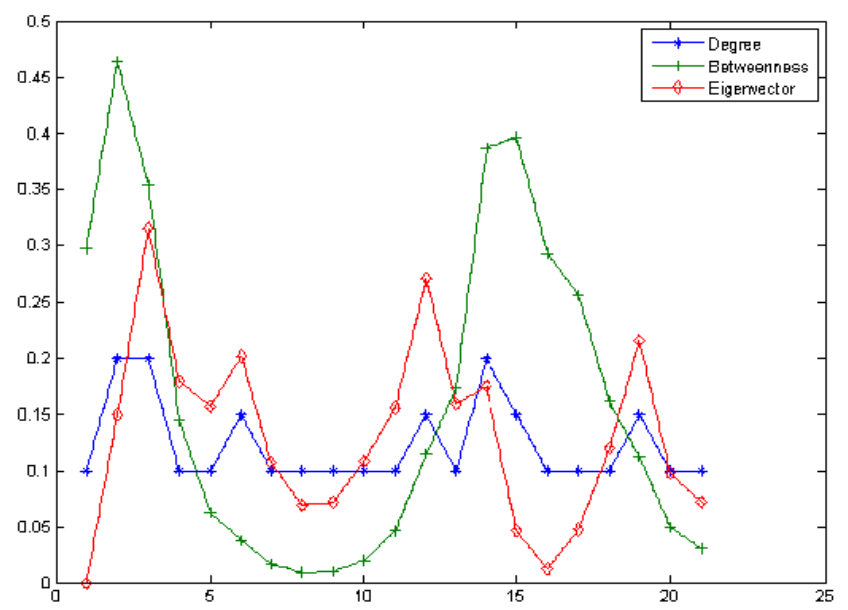

FIGURE II. THE CORRELATION BETWEEN DEGREE, BETWEENNESS AND EIGENVECTORS

In this paper, the FCM clustering method is used to calculate the clustering results as shown in figure III. The three dimensions in figure III represent three clustering factors, node degree, compactness, and vulnerability, where the points represent the distribution of the importance of the nodes. The cross pattern indicates that the node is the key node and the circle indicates that the node is important node, the five-pointed star represents the node as a general node. The key nodes in the figure are nodes 2, 3 and 14, and the important nodes are nodes $1,15,16$ and 17 . The results of clustering and clustering of algorithms in various literatures can prove that the method can effectively evaluate the importance of network nodes.

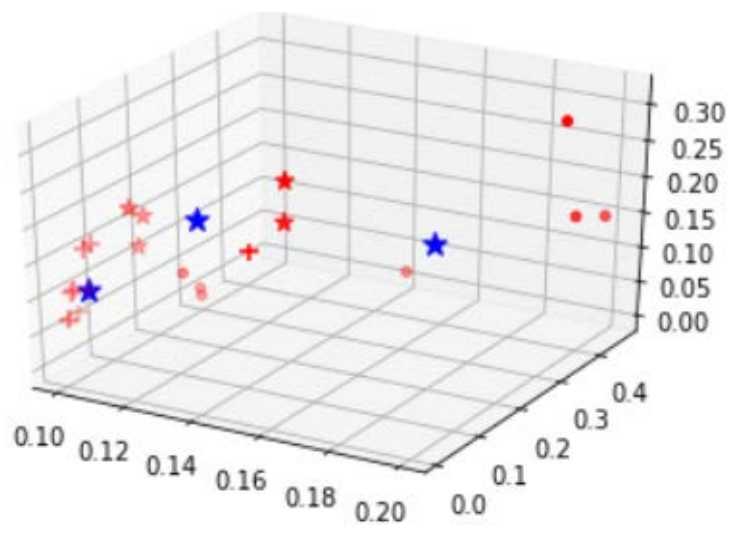

FIGURE III. IMPORTANT CLUSTERING DISTRIBUTION OF NODES

\section{CONCLUSION}

The node importance evaluation of power communication network is an important topic in power communication research. Dealing with the problem that of the previous evaluation indexes for electric power communication networks are relatively single, this paper employs betweenness, degree and eigenvector of the node as the evaluation indexes to evaluate node importance. It uses FCM clustering analysis method to establish the node importance evaluation model for electric power communication networks, and to support the electric power communication network's planning. At the same time, it uses the commonly used network topology model ARPA network to verify the abpve method. This paper proves that this method can effectively evaluates the node importance of power communication networks.

\section{ACKNOWLEDGMENT}

This work is supported by the Science and Technology Project of State Grid Corporation of China:” Integrated Simulation and Service Quality Evaluation of Power Communication Network(XX-71-16-004)”.

\section{REFERENCES}

[1] Zhao Ziyan, Chen Xi, Liu Jianming. Issues of establish a reliable management System in power telecommunication network[J]. Telecommunications for Electric Power System,2006,27(168):58-61.

[2] Xing Ningzhe. Empirical analysis on complex network characteristic of power optical fiber communication network[J]. Optical Communication Technology,2014(3):22-24.

[3] Callaway D S, Newman M E J, Strogatez S H, et al. Network robustne ss and fragility: percolation on random graphs [J]. Physical Review Letters,2000, 85(25):5468-5471.

[4] Tan Yuejin, Wu Jun, Deng Hongzhong. Evaluation Method for Node Importance based on Node Contraction in Complex Networks [J].Systems Engineering-Theory \& Practice,2006,26(11):79-83.

[5] Xiong Jinshi, Li Jianhua, Shen Di, etc. Evaluation Method for Node Importance of Information System Networks Based on Edgebetweenness[J]. Science \& Technology Review,2013,31(14):53-55. 
[6] Chen Yong, Hu Aiqun, Hu Jun, etc. A Method for Finding the Most Vital Node in Communication networks[J].High Technology Letters,2004,14(1):21-24.

[7] Xie Qiongyao, Deng Changhong, Zhao Hongsheng, etc. Evaluation Method for Node Importance of Power Grid Based on the Weighted Network Model[J]. Automation of Electric Power System, 2009,33(4):21-24

[8] Brin S, Page L. The anatomy of a large-scale hyper textual web search engine[J]. Computer Network and ISDN System,1998,30(18):107-117.

[9] Pal N R, Bezdek J C. On cluster validity for the fuzzy c-means model [J]. IEEE Transactions on Fuzzy Systems,3(3):370-379.

[10] Bezdek J C. A physical interpretation of fuzzy ISODATA [J]. IEEE Transactions on System, Man, and Cybernetics,1976,SMC-6,387-389.

[11] Yu Hui, Liu Zun, Li Yongjun. Key nodes in complex networks identified by multi-attribute decision-making method[J]. Acta Physica Sinica,2013,62(2):20204.

[12] Zhou Xuan, Zhang Fengming, Li Kewu, etc. Finding vital node by node importance evaluation matrix in complex networks[J]. Acta Physica Sinica,2012,61(5):050201.

[13] Qin Li, Yang Zi-long, Huang Shu-guang. Synthesis Evaluation Method for Node Importance in Complex Networks[J]. Computer Science,2015,42(2):60-64. 